\title{
Heterogeneous Wireless Access Network Protection for Ultra-Reliable Communications
}

\author{
Elena Grigoreva, Dhruva Shrivastava, \\ Carmen Mas Machuca, Wolfgang Kellerer \\ Chair of Communication Networks \\ Technical University of Munich, Germany \\ (e.grigoreva; dhruva.shrivastava; cmas; wolfgang.kellerer)@tum.de
}

\author{
Juergen Dittrich, Heinz Wilk, \\ Hans-Martin Zimmermann \\ Airbus Defence \& Space, \\ Ulm, Germany \\ name.lastname@airbus.com
}

\begin{abstract}
State-of-the-art wireless communication networks that enable safety and emergency services are predominantly based on Terrestrial Trunked Radio (TETRA). TETRA guarantees four nines of average end-to-end connection availability, but offers low data rate. Originally low-data rate safety and emergency services evolve from basic as voice and messaging towards video and other applications that require high data rate. Emergency and safety-critical voice and messaging, however, fall under a category of ultra-reliable communications and require an average connection availability of five nines, i.e., an average outage time of 5.25 minutes per year. The challenge that we address in this paper is guaranteeing the average connection availability for the ultra-reliable communications as voice, while providing sufficient data rate for the complimentary services as video. We achieve this by using heterogeneous wireless access network, i.e., WLAN, LTE and TETRA, enhanced with automated switching between the wireless technologies through Dynamic Link Exchange Protocol (DLEP). Based on the testbed measurements and reliability analysis, we show that a heterogeneous access network can achieve the five nines availability, while increasing the available data rate in comparison with single technology access networks.
\end{abstract}

\section{INTRODUCTION}

$5 \mathrm{G}$ reopens a plethora of communication challenges such as ultra low delay, e.g., for the Tactile Internet [1], or ultrareliable communications [2] with availability requirements of five nines (i.e., less than six minutes of down time per year). A natural example of ultra-reliable communications are emergency networks, which are used by the public safety organizations like police, fire-fighters and armed forces. For these networks, communication reliability provision is crucial for every end-to-end connection, including the naturally unreliable wireless connection.

Reliable wireless access networks such as Terrestrial Trunked Radio (TETRA) use robust coding and modulation to guarantee communication availability, but sacrificing the data rate [3]. Data rate, delay and reliability requirements to the communication technologies, depend on the application. So far the most common safety application in emergency networks

This work has received funding by the German Research Foundation (DFG) under the grant numbers MA6529/2-1 and KE1863/4-1, and the COST Action CA15127 Resilient communication services protecting end-user applications from disaster-based failures (RECODIS). are voice calls. Voice calls can tolerate up to $3 \%$ packet loss and require a data rate of the range of kbps [4]. Conventional robust wireless technologies as TETRA can satisfy the data rate requirements of the voice and messaging, but not the multimedia services [3]. Thus, a wide range of multimedia applications that could be beneficial in the emergency surroundings as video transmissions and self-updating maps, cannot be supported.

Existing wireless broadband communication technologies, such as LTE or WLAN, enable high data rates. The challenge for these technologies is to ensure the required average connection reliability levels, i.e., five nines [2]. One possible option to increase the reliability is to use multi-path TCP [5], which relies on existing connection diversity. Connection diversity can be achieved by using different wireless links from one technology, e.g., WLAN as in [6]. However, this approach has a number of drawbacks. For example, using the same frequency band and thus sensitivity to the weather conditions.

A prospective way to minimize the influence of the individual technology's drawbacks is to take advantage of heterogeneous networks. Authors in [7] propose integrating LTE and TETRA based on seamless handover between them using Media Independent Handover IEEE 802.21 standard. There are further options to integrate WLAN and TETRA [6], satellite communications and TETRA [8]. A more radical approach would be to design a heterogeneous network, for example through use of a service platform [9] or a multi-tier hierarchal network architecture based on cognitive radio [10]. While all of these approaches have their advantages, they as well share some drawbacks. One of the major drawbacks is the lack of availability analysis. As emergency and safety networks are a part of ultra reliable communications, meeting the reliability requirements is of high importance. Further, a common drawback is a need in adding new network elements to the existing architectures.

In this paper, we conduct a feasibility study on facilitating heterogeneous network interconnection by a radio-to-router protocol without adding extra complex network elements. The feasibility study includes implementation and measurements. We implement Dynamic Link Exchange Protocol (DLEP)based router [11] to switch between the three technologies: WLAN, narrowband LTE and TETRA. In a laboratory testbed 
of WLAN and narrowband LTE, we have measured reliabilityrelevant metrics: packet loss and inter-technology switching time. These measurements are further used for the average wireless link availability calculation for the individual technologies. TETRA characteristics were obtained in previously conducted field trials. To conclude on the proposed architecture suitability for the ultra-reliable communications, we conduct availability analysis. We analyze the impact of the joint use of the heterogeneous communication technologies on the average end-to-end connection availability and verify that ultra-reliable requirements on average system's availability of five nines are satisfied. Thus, the contribution of this paper is twofold: (1) feasibility study and (2) reliability analysis.

The paper's structure reflects the contributions. In Section II, we describe our testbed and introduce the most important implementation details. Section III shows the results of packet loss and switching time measurements for WLAN and narrowband LTE. These packet loss measurements are then used to define the empirical average wireless link availability for the reliability analysis. Section IV presents the reliability analysis. Finally, the paper is wrapped up in Conclusions and Outlook.

\section{Scenario ANd Proposed System Architecture}

Emergency networks differ in technology and application, e.g., dedicated to police or fire fighters, however, they feature common reliability and data rate requirements. In this paper, we assume a scenario, where three communication networks exist in parallel, but do not allow direct traffic routing from one network to another. It can be safely assumed that TETRA network is already deployed; WLAN and LTE are either deployed in parallel to TETRA or used, where available, depending on a concrete scenario.

In particular, we consider an emergency vehicle convoy case. The emergency vehicle convoy consists of several vehicles carrying staff members and network infrastructure to maintain communication between vehicles and back to the command post. Each vehicle carries a full narrowband LTE Infrastructure, i.e., Enhanced Packet Core (EPC) and enhanced Node B (eNB); TETRA Infrastructure, i.e., Digital Switch (DXT) and TETRA Base Station (TBS), and finally WLAN Access Points (AP). This configuration makes it possible for all the vehicles to send data to the command post. Staff members carry Hybrid Terminals (HTs), which support all the wireless access technologies. We upgrade this system architecture with DLEP-enabled router [11] for an automatic change of the wireless access networks. The resulting architecture is summarized in Figure 1.

DLEP is a radio-to-router protocol designed to provide standardized communication between a router and a radio modem. Link layer information from remote radio devices can be used by the router to make decisions on selecting different radio links and to make network convergence faster in the case of mesh networks. DLEP works between the radio and locally connected router only, there is no overhead transported over the radio links. In our laboratory setup, in the dashed frame in Figure 1, we implemented the DLEP specification

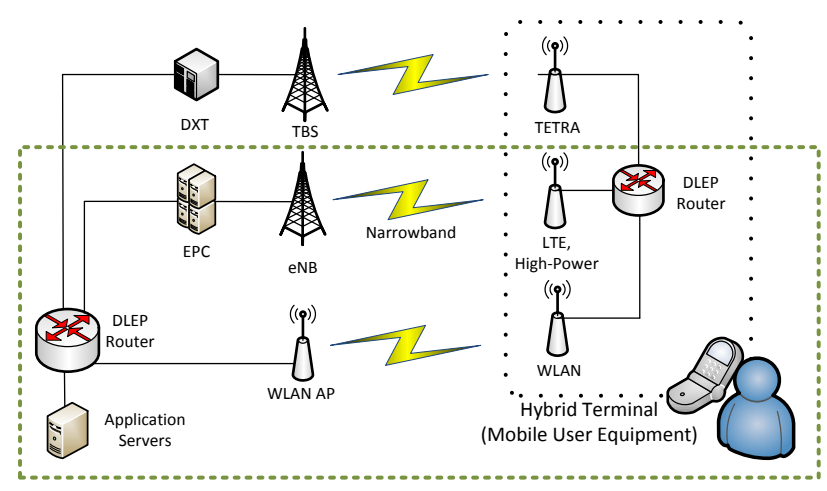

Fig. 1. Proposed system architecture and laboratory prototype (in the dashed frame) for interconnection of the heterogeneous wireless access networks though Dynamic Link Exchange Protocol (DLEP). Narrowband LTE Infrastructure: Enhanced Packet Core (EPC), enhanced Node B (eNB). TETRA Infrastructure: Digital Switch (DXT), TETRA Base Station (TBS). WLAN Access Points (AP).

draft using the open source implementation of Optimized Link State Routing Protocol [12].

The laboratory setup consists of the narrowband LTE infrastructure, WLAN Access Point, High Power LTE Modem and DLEP routers. We performed the measurements on the WLAN and LTE networks.

TABLE I

SYSTEM SPECIFICATIONS

\begin{tabular}{|l|l|}
\hline Parameters & Value \\
\hline Height of Base Station $\left(H_{b}\right)[13]$ & $30 \mathrm{~m}$ \\
\hline Height of User Equipment $\left(H_{m}\right)[13]$ & $1.5 \mathrm{~m}$ \\
\hline \multicolumn{2}{|c|}{ TETRA } \\
\hline Frequency band & $380 \mathrm{MHz}$ \\
\hline TBS Transmit Power [14] & $40 \mathrm{dBm}$ \\
\hline User Equipment Transmit Power [15] & $35 \mathrm{dBm}$ \\
\hline Receiver Sensitivity [15] & $-103 \mathrm{dBm}$ \\
\hline Data Rate & $28.8 \mathrm{Kbps}$ \\
\hline Coverage (from path loss calculation [13]) & $18.78 \mathrm{~km}$ \\
\hline \multicolumn{2}{|c|}{ Narrowband LTE } \\
\hline Frequency band & $400 \mathrm{MHz}$ \\
\hline eNB Transmit Power [16] & $46 \mathrm{dBm}$ \\
\hline High Power Modem Transmit Power [17] & $37 \mathrm{dBm}$ \\
\hline Receiver Sensitivity [18] & $-101.7 \mathrm{dBm}$ \\
\hline Data Rate & $(767.4 \pm 9.84) \mathrm{Kbps}$ \\
\hline Coverage (from path loss calculation [19]) & $18.34 \mathrm{~km}$ \\
\hline \multicolumn{2}{|c|}{ WLAN } \\
\hline Frequency band & $2.4 \mathrm{GHz}$ \\
\hline Access Point Transmit Power [20] & $26 \mathrm{dBm}$ \\
\hline Receiver Sensitivity [18] & $-71 \mathrm{dBm}$ \\
\hline Data Rate & $(19.6 \pm 0.06) \mathrm{Mbps}$ \\
\hline Coverage (from path loss calculation [21]) & $1.44 \mathrm{~km}$ \\
\hline & \\
\hline
\end{tabular}

Table I summarizes the used technology parameters. We list the TETRA parameters as we used in our coverage calculations. Our coverage calculations are based on path loss models. For the TETRA and LTE networks, we used the path loss models from [13] and [19], respectively. In order to obtain comparable coverage of LTE and TETRA, we use high 
power receiver modem [17]. The path loss model to determine coverage of WLAN in the outdoor open area is given in [21].

Note that although we use narrowband LTE to achieve similar coverage as with TETRA, the available data rate with the narrowband LTE is still more than 26 times higher than with TETRA and is sufficient for the chosen services.

\section{Measurements: LTE AND WLAN}

In this Section, we show the results of the measurements on the laboratory prototype for LTE and WLAN. The results for TETRA have been obtained in the field tests. First, we describe the measurement set-up: we state the traffic model and the tools that were used. Then we show the results for the average packet loss measurements. Based on the instantaneous packet loss values and service requirements, we define an empirical wireless link availability. The empirical wireless link availability is later used in our reliability analysis.

Table II summarizes the three UDP traffic cases that have been used in our measurements to cover all the data rate requirements of the emergency services. We assume cyclic traffic of $5 \mathrm{~ms}, 10 \mathrm{~ms}$ or $20 \mathrm{~ms}$ sending cycles. Sending different packet sizes $(32,64,128,256,512$ and 1024 Bytes) at these cycles results in different data rates [22]. We use iPerf [23] to generate the traffic between the transmitter and receiver. To perform the measurements of packet loss using the prototype in the laboratory, Wireshark [24] is used. Note that in our laboratory prototype, we cannot change the distance between the transmitter and receiver up to the coverage limits due to the space limitations.

TABLE II

Measurement TrafFic Characteristics And Data Rates

\begin{tabular}{|l|l|l|l|}
\hline \multirow{2}{*}{ Packet Size } & \multicolumn{3}{|c|}{ Generated Data Rate } \\
\cline { 2 - 4 } & 5 ms Cycle & 10 ms Cycle & 20 ms Cycle \\
\hline 32 Bytes & $0.0512 \mathrm{Mbps}$ & $0.0256 \mathrm{Mbps}$ & $0.0128 \mathrm{Mbps}$ \\
\hline 64 Bytes & $0.1024 \mathrm{Mbps}$ & $0.0512 \mathrm{Mbps}$ & $0.0256 \mathrm{Mbps}$ \\
\hline 128 Bytes & $0.2048 \mathrm{Mbps}$ & $0.1024 \mathrm{Mbps}$ & $0.0512 \mathrm{Mbps}$ \\
\hline 256 Bytes & $0.4096 \mathrm{Mbps}$ & $0.2048 \mathrm{Mbps}$ & $0.1024 \mathrm{Mbps}$ \\
\hline 512 Bytes & $0.8192 \mathrm{Mbps}$ & $0.4096 \mathrm{Mbps}$ & $0.2048 \mathrm{Mbps}$ \\
\hline 1024 Bytes & $1.6384 \mathrm{Mbps}$ & $0.8192 \mathrm{Mbps}$ & $0.4096 \mathrm{Mbps}$ \\
\hline
\end{tabular}

Figures 2, 3 and 4 show the measurement results of the average packet loss for narrowband LTE and WLAN for $5 \mathrm{~ms}, 10 \mathrm{~ms}$ and $20 \mathrm{~ms}$ cycles respectively. From Tables I and II, we observe that narrowband LTE supports data rates up to $800 \mathrm{kbps}$ or respectively packet sizes of less than 512 Bytes for $5 \mathrm{~ms}$ cycle or 1024 Bytes for $10 \mathrm{~ms}$. This is confirmed by results of the average packet loss measurements for narrowband LTE, where we observe an abrupt increase in packet loss after the respected packet sizes. In general, for all the guaranteed data rates the average measured packet loss for the narrowband LTE network and WLAN is less than $1 \%$.

Figure 5 shows an example of the measured instantaneous packet loss for the WLAN and LTE networks for the packet size of 32 Bytes, and the cycle time of $20 \mathrm{~ms}$ over a period of $300 \mathrm{~ms}$. For the same example, the average packet loss as shown in Figure 4 is always lower than $1 \%$. However,

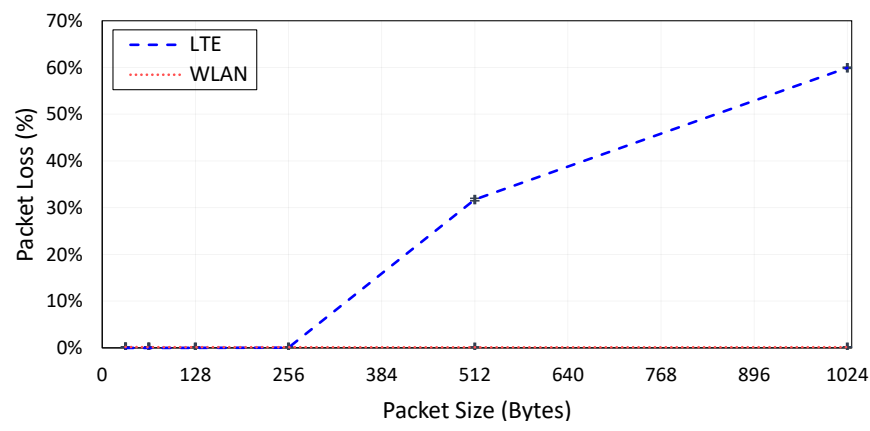

Fig. 2. Average packet loss dependency on the packet size, with $5 \mathrm{~ms}$ generation cycle.

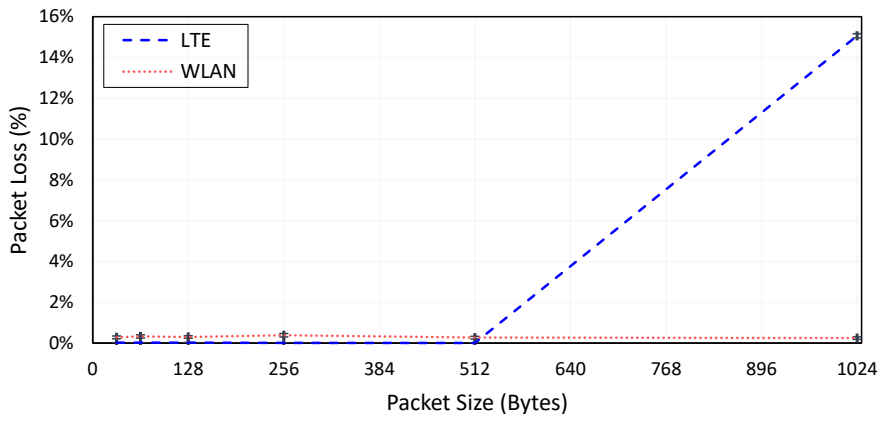

Fig. 3. Average packet loss dependency on the packet size, with $10 \mathrm{~ms}$ generation cycle.

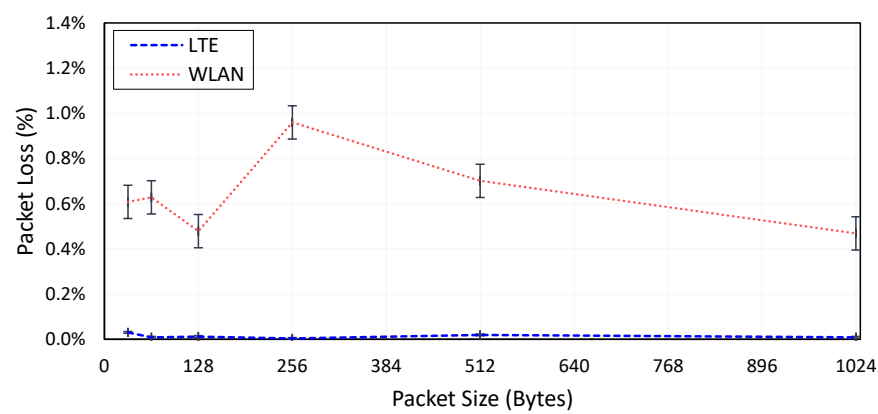

Fig. 4. Average packet loss dependency on the packet size, with $20 \mathrm{~ms}$ generation cycle.

in Figure 5 we observe that there are packet loss bursts. To quantify the wireless channel reliability through packet loss, we define the tolerable packet loss thresholds based on the requirements of the emergency applications. We consider two applications: Critical Safety Messaging (CSM) and Voice over IP (VoIP). For VoIP, we can tolerate packet losses up to $3 \%$ [4], but for the CSM, we can tolerate only up to $1 \%$. With this threshold definition and the measurements example in Figure 5, we conclude that the average packet loss is a metric that is not enough to quantify the wireless link availability. We define the average wireless channel availability as:

$$
\text { Availability }(a)=\frac{T_{\text {Measured }}-\sum_{i=1}^{N} \Delta t_{i}^{\text {Violated }}}{T_{\text {Measured }}},
$$

where $T_{\text {Measured }}$ is the measurement period, which is 3600 seconds ( 1 hour); $\Delta t_{i}^{\text {Violated }}$ is the time when the packet loss violates the given threshold ( $1 \%$ and $3 \%$ ). 


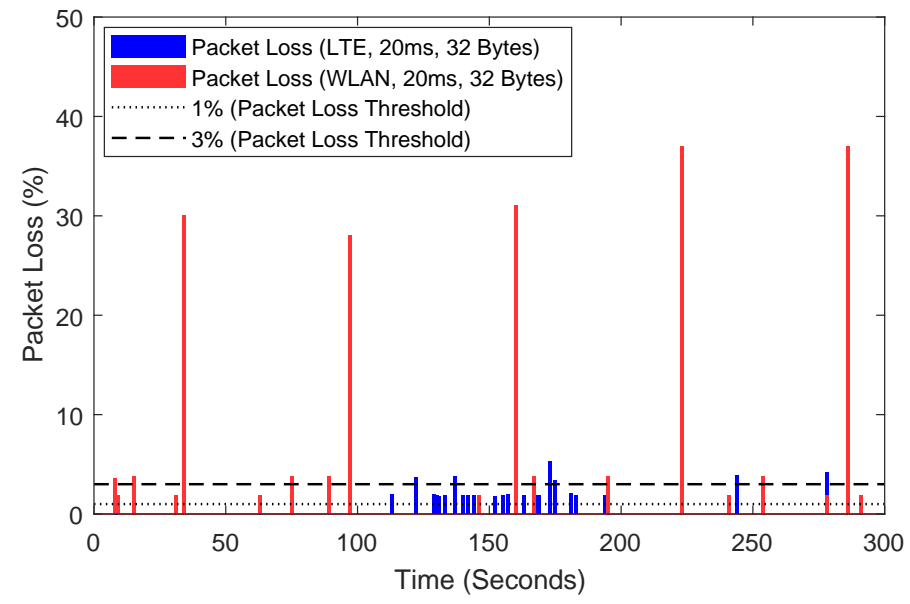

Fig. 5. Example of instantaneous packet loss measurements over $300 \mathrm{~s}$, with $20 \mathrm{~ms}$ cycle and 32 Bytes packet size.

In our measurements, we used different packet sizes and cycle times, see Table II, to cover different types of traffic observed in a packet data network. In order to generalize the measurement results, we average the wireless link availabilities calculated with Eq. (1) of the individual runs for the availability (7200 runs) and for the switching time (64 runs). Table III, summarizes the resulting average availability of the wireless links of LTE and WLAN.

TABLE III

AVERAGE Wireless Link AVAILABILITY

\begin{tabular}{|l|l|l|l|}
\hline \multirow{2}{*}{ Packet Loss } & \multirow{2}{*}{ Application } & \multicolumn{2}{|c|}{ Average availability, \% } \\
\cline { 3 - 4 } & & WLAN & Narrowband LTE \\
\hline $1 \%$ & CSM & 94.000000 & 98.861111 \\
\hline $3 \%$ & VoIP & 97.111111 & 99.555556 \\
\hline
\end{tabular}

Table III shows that both LTE and WLAN individually violate the required availability threshold already at the wireless link level. Thus there is a need in wireless link protection. In this paper, we consider a heterogeneous protection scheme that would allow automated switching from one technology to another in the case of a failure in wireless access network.

If a wireless link failure occurs while data transmission, the transmission shall be switched to the available technology. The time between the failure recognition and data transmission set-up over a functioning wireless access technology, we refer to as "switching time". Table IV, shows the switching time between the LTE and WLAN. To measure the switching time we sent a ping continuously while switching off the working path (LTE or WLAN) so that it switches to the backup path (WLAN or LTE). We observe that the switching times are symmetrical and within $1.5 \mathrm{~s}$ for both directions, when taking the $95 \%$ confidence intervals into account.

TABLE IV

Average SWITCHING Time

\begin{tabular}{|l|l|}
\hline Technology & Average Switching Time, s \\
\hline LTE $\rightarrow$ WLAN & $1.42 \pm 0.27$ \\
\hline WLAN $\rightarrow$ LTE & $1.06 \pm 0.15$ \\
\hline
\end{tabular}

Knowing the switching time is important for wireless link failure definition. A wireless link failure can be defined as an insufficient wireless channel quality over a critical period of time. It is not reasonable to switch to another wireless access technology if the failure time is significantly less than the switching time. Wireless links are influenced by the factors, as weather conditions or interference, which presence is usually limited in time. It makes the wireless link failure definition a challenging and requirement-dependent task. This task, however, is out of the scope of this paper.

\section{Reliability ANALYsis: LTE, WLAN AND TETRA}

In this section, we analyze the average connection availability for our scenario. Unlike fixed networks, where the user location is known at any time, we deal with mobile users, thus we have to consider the user's mobility. We assume that a user has a uniform probability of being anywhere within the system's coverage. The overall average connection availability can be then calculated as:

$$
a_{\text {connection }}=\sum_{i=1}^{N} a_{i} p\left\{d_{i-1} \leq x<d_{i}\right\},
$$

where $N$ is a number of logical regions, $a_{i}$ is the average connection availability in the region $i$, and $p\left\{d_{i-1} \leq x<\right.$ $\left.d_{i}\right\}$ is the probability of finding the user in this region. We have rounded the coverage for TETRA and narrowband LTE to $18.3 \mathrm{~km}$ (see Section II, Table I). So, in this scenario, there are $N=2$ analysis regions as visualized in Figure 6 .

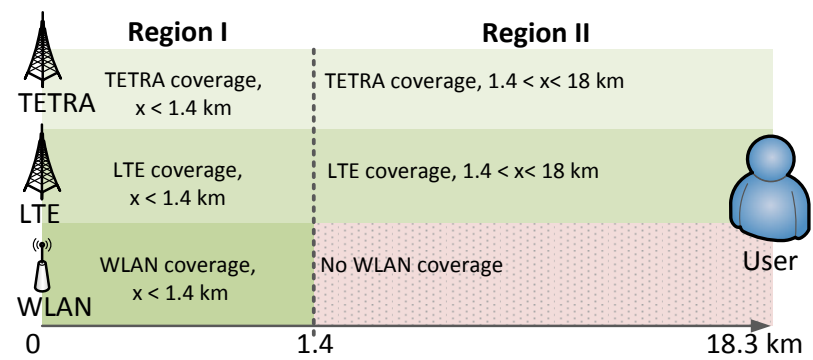

Fig. 6. Two reliability analysis regions: $N=2$, where $x$ denotes user position. Region I: all three technologies WLAN, narrowband LTE and TETRA are available. Region II: only TETRA and narrowband LTE are available.

In region I, all the three technologies (WLAN, LTE and TETRA) are available. This region is defined by the smallest coverage range, i.e., of WLAN or $d_{1}=1.4 \mathrm{~km}$ as shown in Table I. In region II, both, LTE and TETRA, are available. In this case, the region has radius of $d_{2}=18.3-1.4=16,9 \mathrm{~km}$. In this case, there is also a possibility of multi-hop WLAN connection. That is mobile WLAN-capable terminals in an adhoc mode, interconnect so that the entire coverage span can also be covered with WLAN. However, due to low wireless connection availability of WLAN and serial concatenation of the links, this protection option is out of the scope of the paper.

Our average connection availability analysis is based on the Reliability Block Diagrams (RBDs). The average wireless link 
availabilities for WLAN and LTE are defined by the measured packet loss, see Eq. (1) and Table III. The average wireless link availability for TETRA has been obtained in the previous field trials. The average availabilities for the LTE and TETRA core and access network elements as well as for WLAN Access Points (APs) have been obtained from the documentation.

As the DLEP router is a new element, we derive the needed level of availability from the connection availability requirement of five nines. In our study, a hybrid terminal is a user equipment that supports WLAN, LTE and TETRA and has DLEP capabilities. We assume for it the same average availability as for the DLEP router. Table V summarizes the individual average availabilities of all system's components.

TABLE V

INDIVIDUAL AVERAgE AVAILABILITIES FOR WLAN, LTE AND TETRA

\begin{tabular}{|l|l|l|}
\hline Component & Symbol & Average Availability \\
\hline $\begin{array}{l}\text { DLEP Router or Hybrid } \\
\text { Terminal (HT): derived }\end{array}$ & $a_{\text {DLEP }}$ or $a_{\mathrm{HT}}$ & $\geq 0.999996$ \\
\hline \multicolumn{3}{|c|}{ TETRA } \\
\hline $\begin{array}{l}\text { Total: DXT, TBS and } \\
\text { Wireless Link }\end{array}$ & $a_{\text {TETRA }}$ & 0.99998 \\
\hline \multicolumn{3}{|c|}{ Narrowband LTE } \\
\hline EPC & $a_{\mathrm{EPC}}$ & 0.9999616012 \\
\hline eNB & $a_{\mathrm{eNB}}$ & 0.9999448717 \\
\hline \multicolumn{3}{|l|}{ WLAN } \\
\hline Access Point & $a_{\mathrm{AP}}$ & 0.9999948718 \\
\hline
\end{tabular}

In this Section, we show the analysis for the individual protection regions. The Section is concluded by showing the analysis results for the average connection availability over the entire system's coverage.

\section{A. Region I: WLAN, LTE, and TETRA}

Within the coverage radius $d_{1}$ of $1.4 \mathrm{~km}$, WLAN, LTE and TETRA networks work in parallel. Communication traffic can be sent using any technology based on the traffic requirements, e.g., priority, required bandwidth, and network availability. Figure 7 depicts the RBD and Eq. (3) formalizes the average availability for this region.

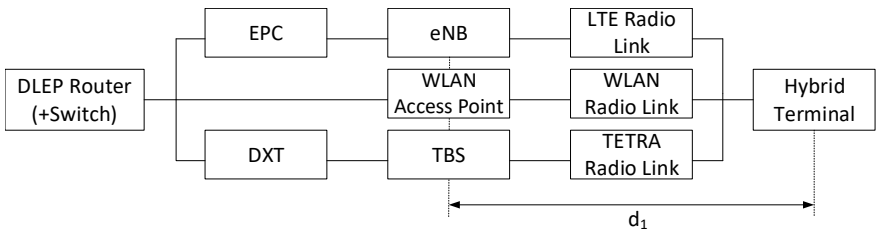

Fig. 7. Reliability Block Diagram of the Region I or $d_{1}=1.4 \mathrm{~km}$, where WLAN, LTE and TETRA are present.

$$
a_{d_{1}}=a_{\mathrm{DLEP}}\left(1-\left(1-a_{\mathrm{TETRA}}\right)\left(1-a_{\mathrm{LTE}}\right)\left(1-a_{\mathrm{WLAN}}\right)\right) a_{\mathrm{HT}},
$$

where $a_{\text {DLEP }}$ and $a_{\mathrm{HT}}$ are obtained through average system availability calculation with a target value of five nines, $a_{\text {TETRA }}$ has been obtained through field trials (see Table V), $a_{\mathrm{LTE}}=$ $a_{\mathrm{EPC}} \cdot a_{\mathrm{eNB}} \cdot a_{\mathrm{WirelessLink}}^{\mathrm{LTE}}$ and $a_{\mathrm{WLAN}}=a_{\mathrm{AP}} \cdot a_{\text {WirelessLink }}^{\mathrm{WLAN}}$. Wireless link availabilities for WLAN and LTE for both reliability critical services, i.e., Critical Safety Messaging (CSM) and
Voice over IP (VoIP), were presented in Table III in the previous section. The rest of the available bandwidth is assumed to be used by the supplementary multimedia traffic, e.g., video. Table VI summarizes the average end-to-end connection availability analysis results for the first region, i.e., for CSM and VoIP.

TABLE VI

Region I: Average ConNection AVAILABILITIES FOR WLAN, LTE AND TETRA INDIVIDUALLY AND FOR HETEROGENEOUS PROTECTION CASE

\begin{tabular}{|l|l|l|}
\hline \multirow{2}{*}{ Technology } & \multicolumn{2}{|l|}{ Average Connection Availability } \\
\cline { 2 - 3 } & CSM & VoIP \\
\hline WLAN & 0.93998766 & 0.97109836 \\
\hline LTE & 0.98851074 & 0.99545448 \\
\hline \multicolumn{3}{|c|}{ Protection } \\
\hline WLAN + LTE & 0.99930307 & 0.999860894 \\
\hline WLAN + TETRA & 0.99999080 & 0.999991422 \\
\hline LTE + TETRA & 0.99999177 & 0.999991909 \\
\hline WLAN + LTE + TETRA & 0.99999199 & 0.999991997 \\
\hline
\end{tabular}

From Table VI, we conclude that the individual WLAN, LTE and TETRA wireless connections cannot guarantee the required average connection availability of five nines. In general, using TETRA allows achieving four nines level of availability, however, only for $28.8 \mathrm{Kbps}$ of traffic. Using LTE and WLAN allows expanding the possible set of services to multimedia. If LTE and WLAN are used with TETRA, both or individually, the required availability of five nines for CSM and VoIP can be achieved. Using LTE and WLAN without TETRA does not achieve even the level of availability of TETRA.

\section{B. Region II: LTE and TETRA}

In this region, LTE and TETRA operate in parallel providing voice and data services. Only LTE can be utilized for the video traffic in the remaining bandwidth. In this case, we consider the distance $d_{2}=18.3 \mathrm{~km}$ as the wireless link has to be available on all the distance to the base station. The RBD of the network for this case is shown in Figure 8 and the average connection availability is given by Eq. (4).

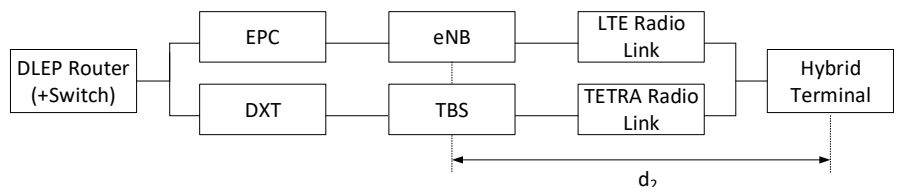

Fig. 8. Reliability Block Diagram of the Region II or $d_{2}=16.6 \mathrm{~km}$, where LTE and TETRA are present.

$$
a_{d_{2}}=a_{\mathrm{DLEP}}\left(1-\left(1-a_{\mathrm{TETRA}}\right)\left(1-a_{\mathrm{LTE}}\right)\right) a_{\mathrm{HT}}
$$

TABLE VII

Region II: Average ConNection Availabilities for LTE AND TETRA INDIVIDUALLY AND FOR HETEROGENEOUS PROTECTION CASE

\begin{tabular}{|l|l|l|}
\hline \multirow{2}{*}{ Technology } & \multicolumn{2}{|l|}{ Average Connection Availability } \\
\cline { 2 - 3 } & CSM & VoIP \\
\hline LTE & 0.98851074 & 0.99545448 \\
\hline \multicolumn{3}{|c|}{ Protection } \\
\hline LTE + TETRA & 0.99999177 & 0.999991909 \\
\hline
\end{tabular}


Table VII summarizes the values of the average availabilities for the CSM and VoIP. We observe, that LTE can compliment TETRA on the entire coverage range providing the required five nines availability and significantly increasing the data rate.

\section{Average Connection Availability}

Applying Eq. (2), we obtain the average connection availability over the entire coverage range. Table VIII summarizes the obtained values with the minimal DLEP router availability of 0.999996, so that the requirement holds.

We further observe, that using WLAN does not significantly influence the availability values. However, WLAN offers high bandwidth and features low cost, thus it can be used as a complimenting technology for providing connection for the applications with lower reliability requirements. Critical VoIP and CSM data shall be transfered over the protection pair of LTE and TETRA.

TABLE VIII

Average Connection Availability over EntiRe Coverage Range

\begin{tabular}{|l|l|l|}
\hline \multirow{2}{*}{ Traffic } & \multicolumn{2}{|c|}{ Average system availability } \\
\cline { 2 - 3 } & WLAN+LTE+TETRA & LTE+TETRA \\
\hline CSM & 0.99999192 & 0.99999177 \\
\hline VoIP & 0.99999179 & 0.99999191 \\
\hline
\end{tabular}

The overall connection availability results are influenced by the user distribution, which is not limited to uniform. Therefore, we have included the individual values for both regions in Tables VI and VII. There are other factors that shall be considered in the future work, for example detecting wireless link failures and deciding, when to switch to a protection technology depending on the switching time as discussed in Section III.

This proof-of-concept study shows that for the ultra reliable communications, as emergency vehicle convoy, using heterogeneous wireless networks as protection allows achieving five nines availability, while increasing the available data rate.

\section{Conclusions And OUtLook}

In this paper, we have investigated the heterogeneous wireless access network protection aiming at achieving five nines availability for ultra-reliable communications. We have looked at an emergency scenario, where WLAN, LTE and TETRA are deployed simultaneously. We have proved our concept that using Dynamic Link Exchange Protocol (DLEP) for automated technology switching achieves the five nines average connection availability, while enhancing the data rate.

There are a number of directions for future investigations. First, the study can be completed with the influence of the distance on the wireless connection availability. Second, there can be investigated a technology switching function, i.e., a clear definition of a wireless failure. Finally, the interference and weather-based disruptions can be evaluated.

\section{REFERENCES}

[1] E. Wong, M. Pubudini Imali Dias, and L. Ruan, "Predictive Resource Allocation for Tactile Internet Capable Passive Optical LANs," Journal of Lightwave Technology, vol. 35, no. 13, pp. 2629-2641, July 2017.
[2] P. Popovski, "Ultra-reliable communication in 5G wireless systems," in 1st International Conference on $5 G$ for Ubiquitous Connectivity, Nov 2014, pp. 146-151.

[3] P. Stavroulakis, "Terrestrial Trunked Radio TETRA: A Global Security Tool, ser. Signals and Communication Technology," Springer, pp. XVI, 302, 2007.

[4] M. N. Dilber, R. Sajjad, and S. Mohammad, "Comparative analysis of traditional telephone and VoIP systems (April , 2014)," IEEE, vol. 12, no. 1, pp. 106-111, 2014.

[5] H. Aida, K. Rojviboonchai, and T. Osuga, "R-M/TCP: protocol for reliable multi-path transport over the internet," in 19th International Conference on Advanced Information Networking and Applications (AINA'05) Volume 1 (AINA papers), vol. 1, March 2005, pp. 801-806.

[6] A. K. Salkintzis, "Evolving public safety communication systems by integrating WLAN and TETRA networks," IEEE Communications Magazine, vol. 44, no. 1, pp. 38-46, Jan 2006.

[7] A. P. Avramova, L. Dittmann, and S. Michail, "MIH based mobility for TETRA-LTE network," in Proceedings of ICT Innovations, 2013.

[8] A. Amditis, A. Oliveira, C. A. Grazia, C. Katsigiannis, D. Kanakidis, E. Sdongos, H. Gierszal, J. Jackson, K. Romanowski, M. Casoni, M. Klapez, N. Patriciello, P. Simplcio, and S. Sonander, "Integration between terrestrial and satellite networks: the PPDR-TC vision," in 2014 IEEE 10th International Conference on Wireless and Mobile Computing, Networking and Communications (WiMob), Oct 2014, pp. 77-84.

[9] A. Pigni, F. Arreghini, P. Danielli, and R. Agrone, "Heterogeneous network testbed for tactical communication in shore scenario," in MILCOM 2015 - 2015 IEEE Military Communications Conference, Oct 2015, pp. 483-488.

[10] B. Canberk, G. A. Shah, O. B. Akan, and O. Ergul, "Adaptive and cognitive communication architecture for next-generation PPDR systems,' IEEE Communications Magazine, vol. 54, no. 4, pp. 92-100, April 2016.

[11] B. Berry, D. Satterwhite, S. Jury, S. Ratliff, and R. Taylor, "InternetDraft Dynamic Link Exchange Protocol (DLEP)," 2017. [Online]. Available: https://tools.ietf.org/html/draft-ietf-manet-dlep-25

[12] H. Rogge. olsrd2,"http://www.olsr.org/". [Online]. Available: http://www.olsr.org/mediawiki/index.php/

[13] M. A. Alamoud and W. Schütz, "Okumura-hata model tuning for TETRA mobile radio networks in saudi arabia," in 2 nd International Conference on Advances in Computational Tools for Engineering Applications (ACTEA), Dec 2012, pp. 47-51.

[14] A. Defense and Space, "Tetra system release 7.0," Tech. Rep.

[15] Hytera, "TETRA hand-held radio," Hytera Mobilfunk GmbH, Bad Münder, Germany, Tech. Rep., 2014. [Online]. Available: http://www.advantec.it/wpcontent/uploads/hytera/pdf/Hytera_TETRA_PT580H_ENG_adv.pdf

[16] Alcatel Lucent, "BB 400 solution LTE practical training," Tech. Rep.

[17] Cassidian Communication GmbH, "HPM description and interfaces," Ulm, p. 16, 2014. [Online]. Available: https://airbusdefenceandspace.com/newsroom/news-and-features/firstcommercially-available-high-power-modem-in-the-400-mhz-spectrumpresented-at-ccw-in-singapore/

[18] National Telecommunications and Information Administration, "The equipment characteristics for LTE FDD transmitters and receivers," Washington, DC, Tech. Rep. [Online]. Available: https://www.ntia.doc.gov/files/ntia/meetings/lte_technical_characteristics.pdf

[19] A. Bavarva, A. Dave, A. Singh, and H. Soni, "MATLAB Simulation Based Various Path Loss Prediction Model," pp. 1157-1160, 2015.

[20] premiertek.net. WLAN Specifications (Long Distance). [Online]. Available: http://www.premiertek.net/products/networking/PL-2712N.html

[21] R. Gandhi, "Empirical Path Loss Model for Outdoor 802.11b Wireless Links," Ph.D. dissertation, IIT Kanpur, 2003. [Online]. Available: http://www.iitk.ac.in/mla/Thesis_Presentation_Draft.pdf

[22] M. Rentschler and P. Laukemann, "Performance analysis of parallel redundant WLAN," in Proceedings of 2012 IEEE 17th International Conference on Emerging Technologies Factory Automation (ETFA 2012), Sept 2012, pp. 1-8.

[23] B. A. Mah, J. Dugan, J. Poskanzer, K. Prabhu, and S. Elliott. iperf3, "https://iperf.fr/". [Online]. Available: https://iperf.fr/

[24] G. Combs. Wireshark, "https://www.wireshark.org/". [Online]. Available: https://www.wireshark.org/ 